SMALL INTESTINE

\title{
Scavenger receptor class B type I (SR-BI) in pig enterocytes: trafficking from the brush border to lipid droplets during fat absorption
}

\author{
G H Hansen, L-L Niels-Christiansen, L Immerdal, E M Danielsen
}

Gut 2003;52:1424-1431

See end of article for authors' affiliations .....................

Correspondence to: Dr E M Danielsen, Department of Medical Biochemistry and Genetics, Panum Institute, Blegdamsvej 3, DK-2200 Copenhagen N, Denmark; midan@imbg.ku.dk

Accepted for publication 9 June 2003
Background: Scavenger receptor class B type I (SR-BI) is known to mediate cellular uptake of cholesterol from high density lipoprotein particles and is particularly abundant in liver and steroidogenic tissues. In addition, SR-BI expression in the enterocyte brush border has also been reported but its role in the small intestine remains unclear.

Aim and methods: To gain insight into the possible function of pig SR-BI during uptake of dietary fat, its localisation in enterocytes was studied in the fasting state and during fat absorption by immunogold electron microscopy and subcellular fractionation.

Results: In the fasting state, SR-BI was mainly localised in the microvillar membrane and in apical invaginations/pits between adjacent microvilli. In addition, a subapical compartment and small cytoplasmic lipid droplets were distinctly labelled. During lipid absorption, the receptor was found in clathrin positive apical coated pits and vesicles. In addition, cytoplasmic lipid droplets that greatly increased in size and number were strongly labelled by the SR-BI antibody whereas apolipoprotein A-1 positive chylomicrons were largely devoid of the receptor.

Conclusion: During absorption of dietary fat, SR-BI is endocytosed from the enterocyte brush border and accumulates in cytoplasmic lipid droplets. Internalisation of the receptor occurs mainly by clathrin coated pits rather than by a caveolae/lipid raft based mechanism.
S cavenger receptor class B type I (SR-BI) belongs to the CD36 family of cell surface receptors ${ }^{1-3}$ and was initially identified as a scavenger receptor for acetylated low density lipoprotein (LDL) in CHO cells. ${ }^{4}$ It was soon found to bind to several other ligands, including oxidised and unmodified LDL and very low density lipoprotein (VLDL), but its physiologically relevant ligand is now thought to be high density lipoprotein (HDL) to which it binds with high affinity. ${ }^{15}$ Its main function as a HDL receptor is indicated by high SR-BI expression in the liver and steroidogenic tissues which also have the highest level of selective uptake of cholesteryl esters from HDL. ${ }^{2}$ In addition, a HDL receptor function is implied by the coordinately regulated expression of SR-BI with cholesterol metabolism in steroidogenic tissues in response to trophic hormones. Finally, altering SR-BI activity by overexpression or gene knockout lowers and raises plasma levels of HDL cholesterol, respectively. ${ }^{16}$

SR-BI is generally thought to function as a non-endocytic receptor and promotes selective uptake of cholesteryl ester from the lipoprotein, independent of apolipoproteins. The precise molecular mechanism whereby this occurs is as yet poorly understood but SR-BI is believed to act not only as a docking site for the HDL particle but also as a direct facilitator of the specific transfer of lipid to the cell. According to a proposed model, cholesteryl ester diffuses down a concentration gradient from the core of the docked HDL particle to the cell plasma membrane via a non-aqueous pathway. ${ }^{7}$ Conceivably, this may occur by the ability of SR-BI to form some kind of channel for the cholesteryl ester from which water is excluded. The function of SR-BI may depend on its localisation in caveolae/lipid raft microdomains ${ }^{8}{ }^{9}$ from where HDL cholesteryl ester transport to internal membranes occurs as part of a caveolin-annexin A2 lipid-protein complex. ${ }^{10}$ However, in contrast with this accepted view of SR-BI functioning as a cell surface receptor, it has also been reported to be an endocytic receptor that mediates HDL particle uptake and recycling, but not degradation. ${ }^{11}$

In the small intestine, SR-BI has been found in the brush border membrane of enterocytes ${ }^{12-14}$ raising the possibility that it plays a role in dietary/biliary cholesterol absorption. ${ }^{15-17}$ In the present work, we studied the SR-BI in pig small intestine in the fasting state, during fat absorption, and in cultured mucosal explants under conditions mimicking fat absorption in vivo. In the brush border, the receptor was mainly localised in the lower part of the microvilli and in invaginations between adjacent microvilli. Intracellularly, it was seen in a subapical compartment and, most prominently, in small cytoplamic lipid droplets, whereas multivesicular bodies/lysosomes were devoid of labelling. Surprisingly, microvillar SR-BI was not associated with lipid rafts, and during the fat absorptive process it localised in apical clathrin coated pits and vesicles. Intracellularly, it accumulated in newly formed lipid droplets.

\section{METHODS}

\section{Reagents and antibodies}

Rabbit polyclonal antibodies to a peptide containing residues 496-509 from mouse SR-BI were manufactured by Novus Biologicals (Littleton, Colorado, USA) and swine antirabbit IgG by Dako (Glostrup, Denmark). A monoclonal antibody to rat clathrin heavy chain was from Transduction Laboratories (Lexington, Kentucky, USA), and rabbit antibodies to apolipoprotein A-1 were those previously described..$^{18}$ Gold

\footnotetext{
Abbreviations: SR-BI, scavenger receptor class B type I; LDL, low density lipoprotein; VLDL, very low density lipoprotein; HDL, high density lipoprotein; SDS-PAGE, sodium dodecyl sulphate-polyacrylamide
} gel electrophoresis 
labelled goat antirabbit and goat antimouse antibodies were from Pharmacia Amersham Biotech (Little Chalfont, UK). Bovine bile and pancreatin from porcine pancreas (containing a mixture of digestive enzymes) were obtained from Sigma (St Louis, Missouri, USA). Cholesterol was purchased from ICN Biomedicals Inc. (Aurora, Ohio, USA).

Jejunal segments of pig small intestine, taken l-2 m from the pylorus, were kindly provided by Letty Klarskov and Mette Olesen from the Department of Experimental Medicine, Panum Institute, Copenhagen, Denmark. Unless otherwise stated, the small intestines used were taken from animals that had been fasted overnight. In one experiment, a pig was fed $250 \mathrm{~g}$ of fat (butter) three hours prior to surgery.

\section{Organ culture of mucosal explants}

After rinsing of the small intestine in Hanks' buffered salt solution, mucosal explants obtained from two animals were excised and cultured in NEM medium for periods of up to one hour. ${ }^{19}$ In experiments with fat absorption, a solution of corn oil $(10 \%)$, cholesterol $(0.5 \%)$, bile $(1 \%)$, and pancreatin ( $1 \%)$ was made up in NEM medium, sonicated for one minute, and preincubated for one hour at $37^{\circ} \mathrm{C}$ before it was added to the mucosal explants.

\section{Electron microscopy}

Postembedding immunogold labelling of ultrathin Epon sections, using antibodies to SR-BI, was performed as previously described. ${ }^{20}$

For immunogold labelling of ultracryosections, mucosal segments were fixed in $2 \%$ paraformaldehyde and $0.1 \%$ glutaraldehyde for two hours at $4^{\circ} \mathrm{C}$ or in $3 \%$ paraformaldehyde and $2 \%$ glutaraldehyde for 15 minutes at $4^{\circ} \mathrm{C}$. Ultracryosectioning and immunogold labelling using antibodies to SR-BI, apolipoprotein A-1, and clathrin heavy chain were performed as previously described. ${ }^{21}{ }^{22}$ Sections were examined under a Zeiss EM 900 electron microscope equipped with a Mega View II camera system.

Control experiments were performed by omission of the primary antibody, and in immunogold double labelling experiments the second primary antibody was omitted. Essentially no unspecific labelling was observed in controls.

For morphometric analysis, the number of coated pit-like structures at the apical cell surface of enterocytes from the lower to the mid villus region was counted in 50 randomly chosen apical views from explants, cultured under the above fat absorption conditions for 0 minutes (controls) and 30 minutes, respectively.

\section{Preparation of membrane fractions, immunopurification, and lipid raft analysis of SR-BI} Microvillar and $\mathrm{Mg}^{2+}$ precipitated membranes were prepared from uncultured intestinal mucosal scrapings of fasted animals by the divalent cation precipitation method, ${ }^{23}$ as described previously. ${ }^{24}$ The protease inhibitors leupeptin and aprotinin were included in all buffers $(10 \mu \mathrm{g} / \mathrm{ml})$. Briefly, scrapings were homogenised in $2 \mathrm{mM}$ Tris $\mathrm{HCl}, 50 \mathrm{mM}$ mannitol, pH 7.1, using a manually operated Potter-Elvehjem homogeniser. The homogenate was cleared by centrifugation at $500 \mathrm{~g}, 10$ minutes, and $\mathrm{MgCl}_{2}$ was added to a final concentration of $10 \mathrm{mM}$. After incubation on ice for 15 minutes, the preparation was centrifuged at $1500 \mathrm{~g}$, 10 minutes, to pellet intracellular and basolateral membranes. Finally, the supernatant was centrifuged at $48000 \mathrm{~g}$, one hour, to obtain a pellet of microvillar membrane vesicles and a supernatant of soluble proteins.

For immunoisolation of SR-BI, microvillar membranes were resuspended and solubilised for 15 minutes at $20^{\circ} \mathrm{C}$ by $1 \%$ Triton X-100 in $25 \mathrm{mM}$ HEPES-HCl, $150 \mathrm{mM} \mathrm{NaCl}$, $\mathrm{pH} 7.1$, in the presence of protease inhibitors. Extracts were cleared by centrifugation at $20000 \mathrm{~g}, 10$ minutes, and preincubated with protein A-Sepharose for one hour, followed by incubation with SR-BI antibodies coupled to protein A-Sepharose for two hours. A control sample was incubated in parallel with protein A-Sepharose without SRBI antibodies. The protein A-Sepharose beads were washed three times with buffer before analysis by sodium dodecyl sulphate-polyacrylamide gel electrophoresis (SDS-PAGE).

Lipid raft analysis of the membrane fractions was performed by Triton X-100 extraction (1\%) on ice followed by sucrose gradient centrifugation, ${ }^{25}$ as previously described, ${ }^{26}$ with the modification that the extracts were placed in a $60 \%$ sucrose cushion with a 50-25\% sucrose gradient layered on top.

\section{Electrophoresis and immunoblotting}

SDS-PAGE was performed in 10\% polyacrylamide gels. ${ }^{27}$ After electrophoresis and transfer onto Immobilon membranes, immunoblotting was performed with antibodies to SR-BI (dilution 1:2000-4000). Blots were developed by an electrochemiluminescence detection reagent according to the protocol supplied by the manufacturer (Amersham Pharmacia Biotech).

\section{RESULTS}

SR-BI localisation in the enterocyte in the fasting state Figure 1 shows localisation of SR-BI in the enterocyte by immunogold electron microscopy performed on ultracryosections. Overall, labelling was primarily observed in the apical region of the cell. In the brush border, SR-BI was seen over the microvilli but the receptor was also frequently observed at the base of the microvilli and in invaginations in the apical membrane between adjacent microvilli (fig 1A). In the apical cytoplasm, the receptor was detected in endosomal tubulovesicular structures (fig 1B), often referred to as the subapical compartment. ${ }^{28}$ In addition, small dense cytoplasmic bodies that we take to represent lipid droplets were intensely labelled by the SR-BI antibody whereas multivesicular bodies/lysosomes were devoid of any labelling (fig 1C, D).

\section{SR-BI localisation during fat absorption}

Figure 2 shows the supranuclear region of enterocytes three hours after ingestion of a fatty meal. Morphologically, the fat absorptive state is at first characterised by the appearance of numerous lipid inclusions in the cytoplasm. As previously described by others, ${ }^{29}$ some of these were relatively light, and often seen in clusters, whereas others, darker and usually larger in size, also appeared in the cytoplasm. Of these, only the latter type of lipid droplets were conspicuously labelled by the SR-BI antibody (fig 2B). As can be seen, not only the rim but also the interior of the droplets were labelled, indicating that some of the SR-BI becomes entirely immersed in the lipid phase. The lighter lipid droplets not labelled by the SRBI antibody were labelled with antibodies to apolipoprotein A-1 (fig 2C), as previously described. ${ }^{18}$ They thus represent nascent chylomicrons en route through the secretory pathway to the lateral cell surface from where exocytosis takes place. 3031

Taken together, the results show that the lipid droplets positive for SR-BI are those transiently accumulated in the cytosol and not chylomicrons awaiting exocytosis. Importantly, this selectivity of labelling by the SR-BI antibody is also evidence that the antibody does not bind to lipid droplets in a non-specific way.

\section{Internalisation of SR-BI during fat absorption}

To study in closer detail the early stages of fat absorption, mucosal explants were cultured for periods up to 60 minutes in the presence of a mixture of corn oil, cholesterol, bile, and 

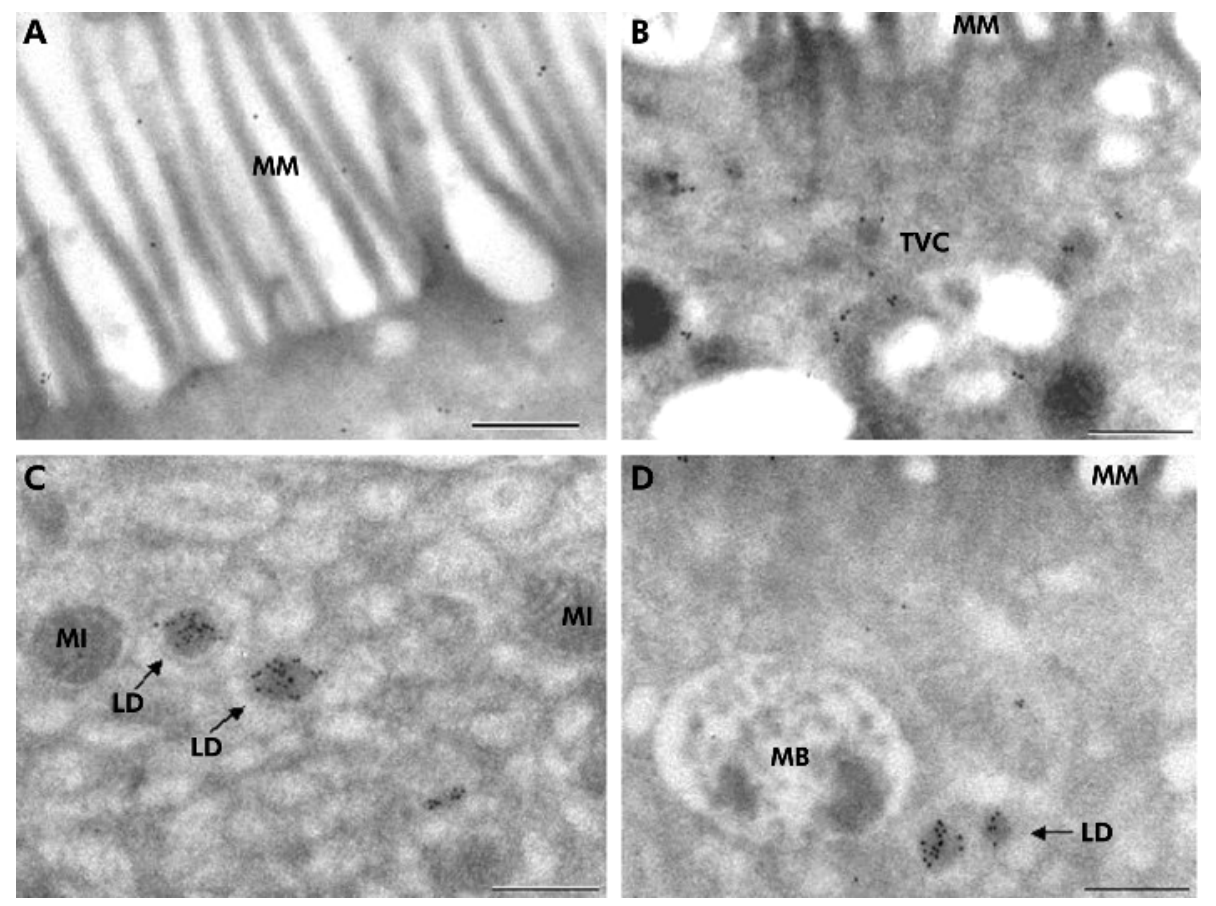

Figure 1 Localisation of scavenger receptor class B type I (SR-BI) in the enterocyte in the fasting state. In the brush border, immunogold labelling was seen over the microvilli as well as at the base of the microvilli (MM) and in invaginations between adjacent microvilli (A). In the cytoplasm, labelling was seen in an apical tubulovesicular compartment (TVC) (B), and in small lipid droplets (LD) (C, D). Multivesicular bodies/lysosomes (MB) and mitochondria (MI) were not labelled (D). Bars $0.5 \mu \mathrm{m}$.

pancreatin. By 15 minutes, many coated pits appeared at the apical surface of enterocytes (fig 3A), and by morphometric analysis of 50 randomly chosen views the number of coated pit-like structures increased from 19 in controls to 88 in explants, cultured for 30 minutes. The coated pits were labelled sparsely but specifically by an antibody to the heavy chain of rat clathrin (fig 3B), and double immunogold labelling for SR-BI and clathrin confirmed that the coated pits internalising the receptor were indeed of the clathrin coated type (fig 3C, D). Interestingly, nascent lipid droplets that labelled for SR-BI could be observed in the membrane of the endoplasmic reticulum (fig 4A). For comparison, fig 4B shows immunogold labelling of the same apical region of the enterocyte for the brush border enzyme aminopeptidase $\mathrm{N}$ during fat absorption. The enzyme localised at the apical surface and in apical tubulovesicular membranes as previously described ${ }^{32}$ but importantly, the SR-BI positive lipid droplets were devoid of labelling. This experiment shows that SR-BI trafficking to the lipid droplets is not a general pathway taken by other membrane proteins from the apical surface during fat absorption. In addition, it demonstrates that labelling of lipids droplets, observed with the SR-BI antibody, is not due to unspecific binding to the lipid of the primary rabbit antibody or the secondary gold conjugated antibody.

\section{SR-BI localisation by subcellular fractionation}

As shown by immunoblotting, the $82 \mathrm{kDa}$ polypeptide of SR-BI was prominently present in the microvillar fraction of enterocytes (fig 5A), in agreement with its subcellular distribution seen by immunogold electron microscopy. In addition, SR-BI was also seen in the $\mathrm{Mg}^{2+}$ precipitated fraction, a bulky fraction consisting of various non-microvillar membranes, including intracellular membranes, whereas the soluble fraction was devoid of the $82 \mathrm{kDa}$ polypeptide. Figure 5B shows that the antibody used for immunogold electron microscopy and immunoblotting was also capable of distinctly immunoprecipitating the $82 \mathrm{kDa}$ polypeptide of SR-BI, thus further demonstrating its specificity.

\section{Lipid raft association of SR-BI}

Figure 6 shows a lipid raft analysis by sucrose gradient centrifugation ${ }^{25}$ of SR-BI from $\mathrm{Mg}^{2+}$ precipitated and microvillar membrane fractions of enterocytes. SR-BI from the $\mathrm{Mg}^{2+}$ precipitated membrane fraction was partially localised in the floating fractions, indicating an association with lipid rafts (fig 6A). In contrast, in microvillar membranes, prepared from the same homogenate and analysed in parallel, the receptor was exclusively present in the nonfloating part of the gradient, indicating that it is not associated with lipid raft microdomains in microvilli (fig $6 \mathrm{~B})$. For comparison, the distribution in the same gradient of a marker for lipid rafts, galectin 4 , as well as a marker for "non-rafts", lactase-phlorizin hydrolase, ${ }^{33}$ is shown, demonstrating that the two microvillar subfractions were effectively separated in the experiment (fig 6B). The surprising non-raft localisation of microvillar SR-BI was further analysed in an experiment that did not involve an overnight centrifugation where dissociation of the SR-BI from lipid rafts conceivably might occur (fig 7). Here, the receptor was almost entirely present in the non-raft (NR) fraction, in common with lactase, regardless of whether cholesterol was extracted from the microvillar membranes by methyl- $\beta$-cyclodextrin prior to solubilisation with detergent. In contrast, galectin-4 remained in the lipid raft fraction (R) after cholesterol extraction, in agreement with our previous observation that microvillar lipid rafts are cholesterol independent and seem instead to rely on a high content of glycosphingolipids. ${ }^{33} 34$

In other cell types, SR-BI has previously been reported to be present in caveolae, ${ }^{8}$ which are composed of lipid rafts, ${ }^{35}$ but clearly the receptor residing in the microvillar membrane of the enterocyte does not interact with the lipid raft microdomains of this highly specialised cell membrane. ${ }^{36}$ 

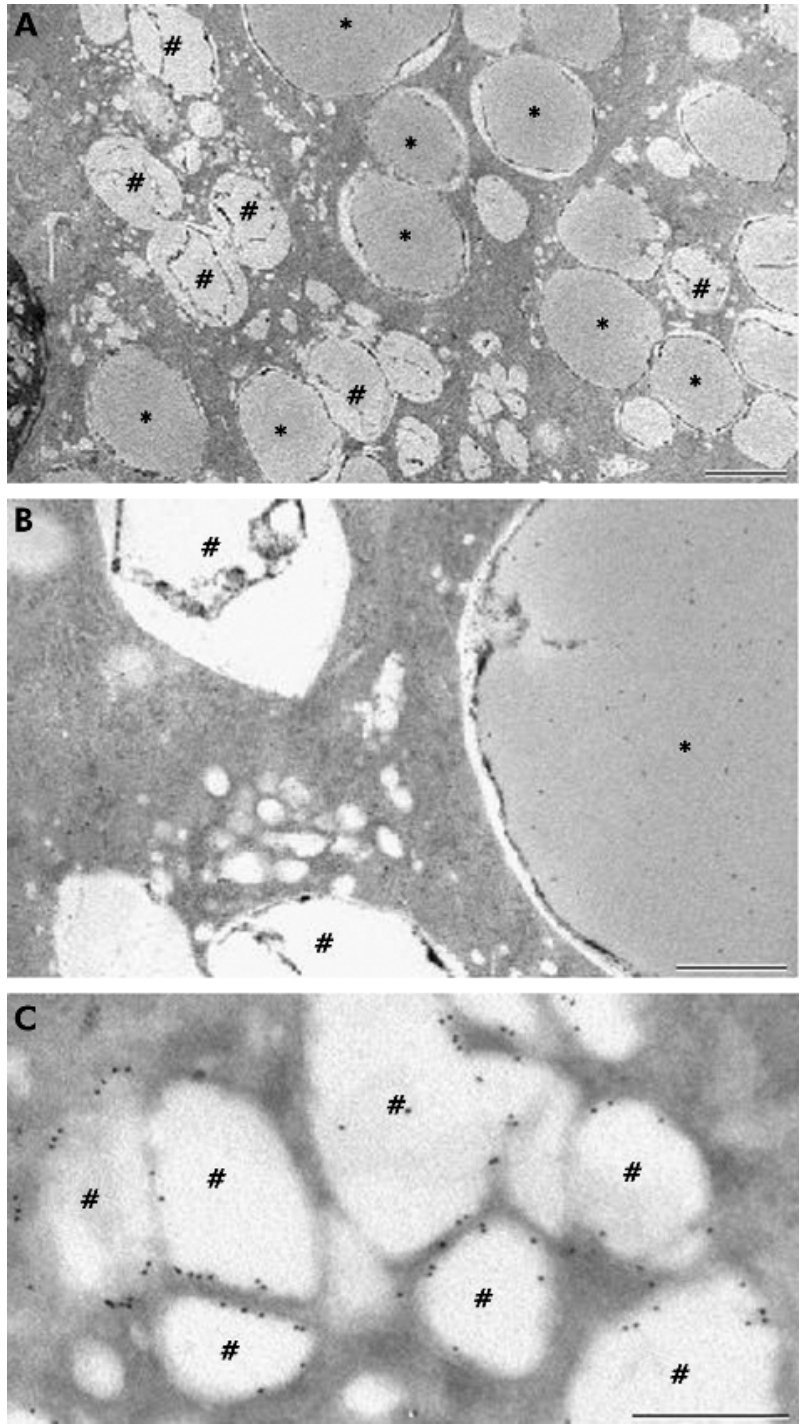

Figure 2 Localisation of scavenger receptor class B type I (SR-BI) in large cytoplasmic lipid droplets during fat absorption. (A) Electron micrograph of an Epon section showing numerous lipid droplets in the cytoplasm. Two morphologically distinct types of lipid droplets were seen: some relatively large and dark (*) and some relatively small and light, often seen in clusters (\#). (B) Immunogold labelling of an Epon section for SR-BI, showing that only the large and dark lipid droplets were labelled. Note that labelling was not confined to the rim but distributed over the entire droplet. (C) Double immunogold labelling of an ultracryosection for apolipoprotein A-1 (10 nm particles) and SR-BI (5 nm particles) of a cluster of the small and light type of lipid droplets. Apolipoprotein A-1 was localised to the rim of the droplets, thereby identifying them as nascent chylomicrons. SR-BI labelling was not seen in chylomicrons. Bars $2 \mu \mathrm{m}(\mathrm{A}) ; 1 \mu \mathrm{m}(\mathrm{B}) ; 0.2 \mu \mathrm{m}(\mathrm{C})$.

\section{DISCUSSION}

SR-BI is now widely believed to function physiologically as a HDL receptor, facilitating the cellular uptake of cholesterol, mainly in the form of cholesteryl esters. ${ }^{915}$ Unlike the classical LDL receptor however, SR-BI has mainly been characterised as a non-endocytic receptor that mediates selective uptake of cholesteryl esters from HDL by a mechanism that does not involve internalisation of the lipoprotein particle. Although expression of SR-BI is highest in the liver and steroidogenic tissues, the receptor is found in many other tissues and cells, including the enterocytes of the small intestine. ${ }^{13}$ In the present work, we investigated the subcellular localisation of SR-BI in small intestinal enterocytes with particular emphasis on its trafficking during dietary lipid absorption. Conceivably, SR-BI present at the lumenal surface of enterocytes, previously demonstrated by immunocytochemistry and blotting, ${ }^{17}$ could be suggestive of an additional role for the receptor as a mediator of dietary/ biliary cholesterol absorption. This hypothesis lends support to reports that intestinal cholesterol absorption is protein mediated $^{37}$ and exhibits saturation kinetics. ${ }^{38}$ In addition, it can be inhibited by cholesterol analogues ${ }^{39}$ and HDL and apolipiprotein A-1. ${ }^{12}$ However attractive this notion is, the observation that cholesterol absorption is not reduced and faecal sterol excretion not increased in SR-BI knockout mice ${ }^{40}$ argues against the receptor playing an essential role in intestinal cholesterol absorption.

Despite the uncertainty concerning the physiological ligand(s) for SR-BI in the gastrointestinal tract, it is reasonable to assume that the receptor must be active during absorption of dietary lipid, and the main finding of the present work was that intestinal SR-BI undergoes endocytosis during fat assimilation in the enterocyte. The evidence supporting this conclusion is based on the observation concerning the localisation and trafficking of the receptor. Firstly, in the brush border, SR-BI frequently resides at the base of the microvilli. Secondly, the receptor is prominently present in coated membrane pits/invaginations between adjacent microvilli. Due to the dense and rigid microvillar cytoskeleton, these patches of apical membrane are the only accessible sites for endocytic membrane traffic from the brush border. Thirdly, SR-BI labelling in the tubulovesicular membranes, also known as the subapical compartment, is consistent with receptor trafficking between the apical surface and interior of the enterocyte. Fourthly, SR-BI accumulates in cytoplasmic lipid droplets. Labelling of the small scattered droplets seen in the fasting state was distinct but the huge increase seen during fat assimilation strongly indicates that lipid droplets are the target compartment for internalised SR-BI. This conclusion is supported by the observation that no labelling was observed in multivesicular bodies/lysosomes, showing that internalisation of the receptor is not part of a degradative pathway.

We find localisation of SR-BI not only at the rim but also within the core of the lipid droplets, seen both in Epon and in ultracryo sections, particularly intriguing. The specificity of the antibody demonstrated both by immunoblotting and purification strongly argues against unspecific staining, and equally important, the lipid core of chylomicrons was not labelled by the SR-BI antibody, ruling out unspecific staining of triacylglycerol. An explanation for such an unusual behaviour of a membrane glycoprotein could lie in the unique lipophilic properties of the extracellular domain of the receptor. On binding to a HDL particle at the cell surface, SR$\mathrm{BI}$ has been proposed to form a non-aqueous channel allowing cholesteryl ester from the core of the lipoprotein to diffuse down a concentration gradient to the cell membrane. ${ }^{7}$ In the light of such a model, we find it conceivable that SR-BI, when associating with a cytoplasmic lipid droplet enveloped by a lipid monolayer, undergoes a conformational change that allows it to partition into the non-aqueous phase.

The notion that SR-BI, contrary to prevailing opinion, can function as an endocytic receptor has previously been reported. ${ }^{11}$ Thus in a number of cell types (CHO cells, hepatocytes, MDCK cells), a SR-BI mediated internalisation of HDL has been described. ${ }^{3}$ In epithelial cells, HDL constituents undergo selective sorting with recycling of HDL protein from the basolateral plasma membrane and transcytotic secretion of cholesterol from the apical surface. That SR-BI may indeed act as an endocytic receptor is also supported by identification of a PDZ domain protein, CLAMP, 

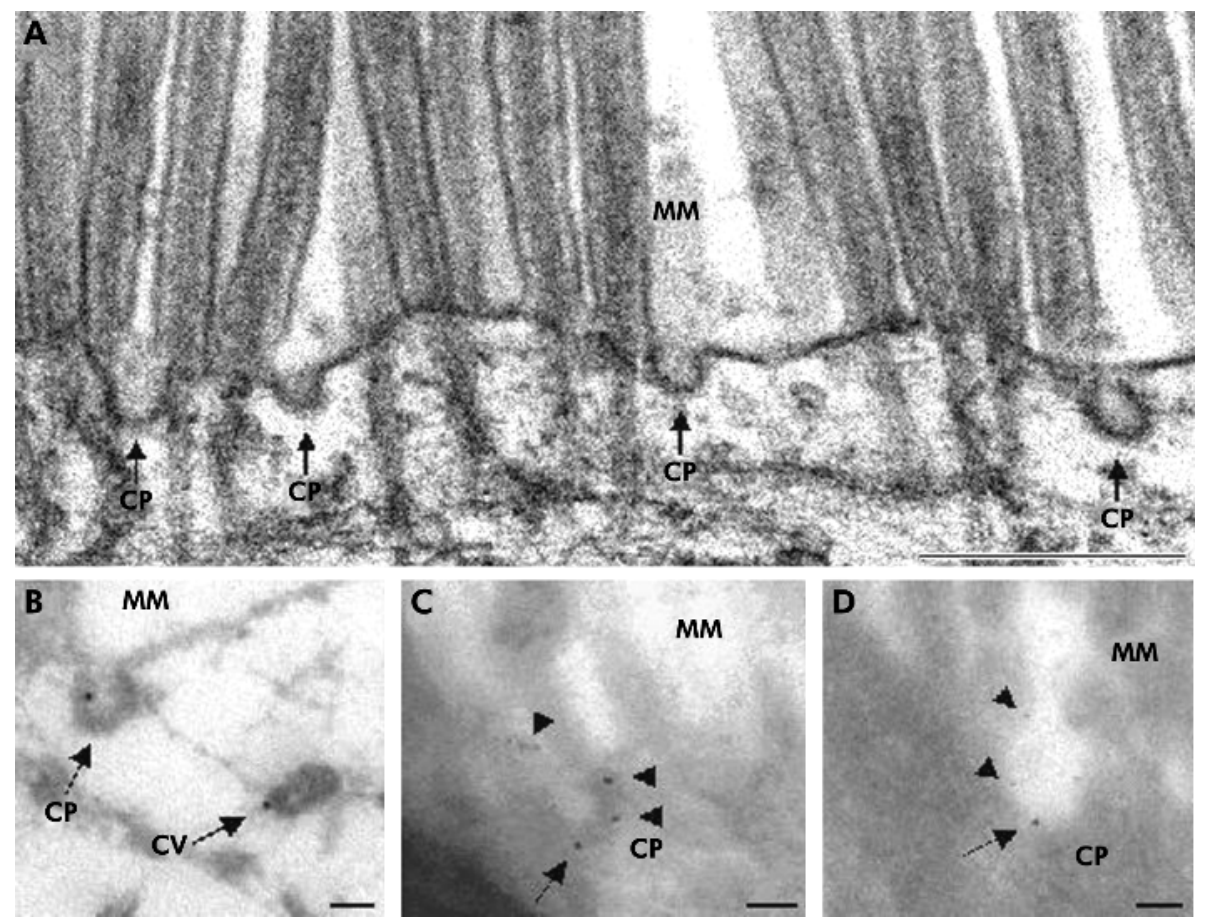

Figure 3 Scavenger receptor class B type I (SR-BI) localisation in coated pits during the early phase of lipid absorption. (A) Electron micrograph of the brush border region of an enterocyte of an explant, cultured for 30 minutes in the presence of lipid, as described in the methods section. Many coated pits (CP) are present between adjacent microvilli (MM) in the terminal web region. (B) Immunogold labelling of an ultracryosection for clathrin, showing labelling of an apical coated pit (CP) and a coated vesicle (CV). (C, D) Double immunogold labelling of ultracryosections for SR-BI $(5 \mathrm{~nm}$ particles, indicated by arrowheads) and clathrin (10 nm particles, indicated by arrows), showing colocalisation in coated pits at the apical surface. Bars $0.5 \mu \mathrm{m}$ (A); $0.2 \mu \mathrm{m}(\mathrm{B}-\mathrm{D})$.
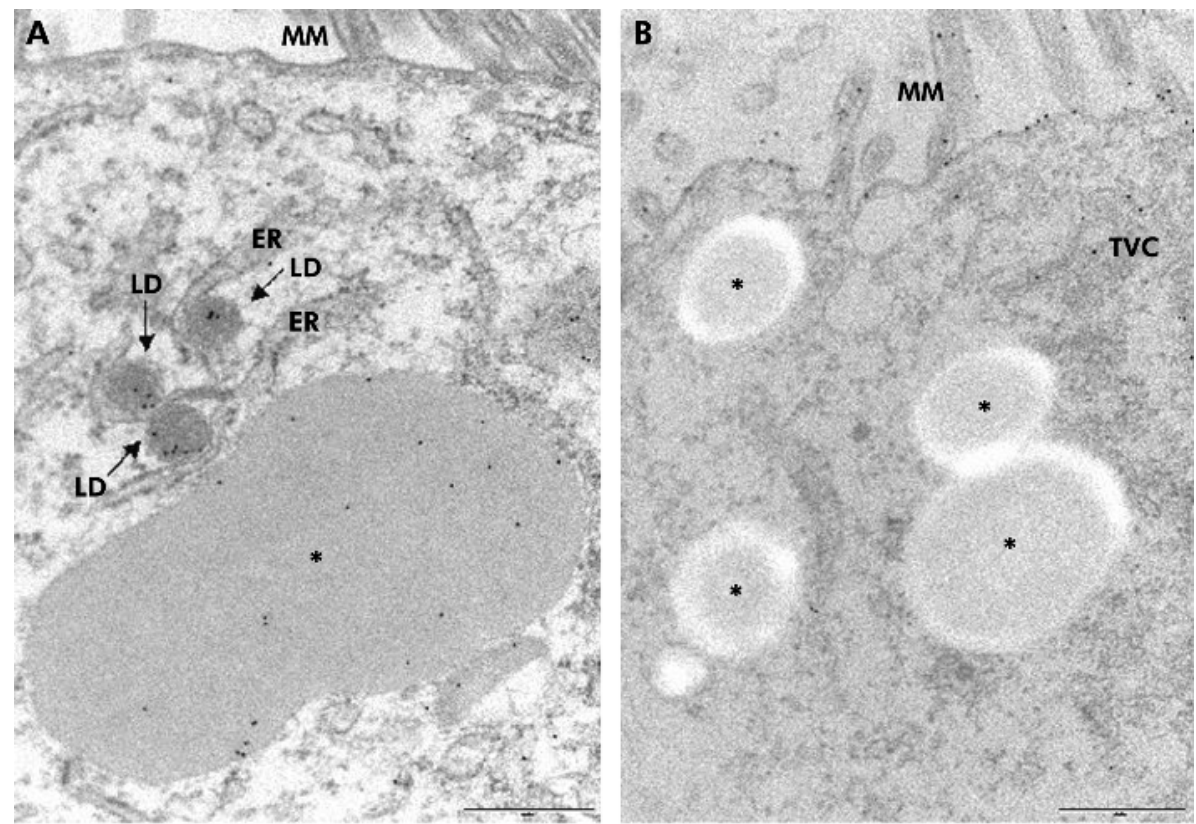

Figure 4 Scavenger receptor class B type I (SR-BI) localisation in nascent lipid droplets during the early phase of lipid absorption. (A) SR-BI immunogold labelling of an Epon section from a mucosal explant, cultured for 60 minutes in the presence of lipid. Labelling was seen in nascent lipid droplets (LD), associated with the endoplasmic reticulum (ER), as well as in a large cytoplasmic lipid droplet (*). (B) Immunogold labelling for aminopeptidase $\mathrm{N}$ in a similar Epon section. The enzyme was seen at the microvillar surface $(M M)$ and in the apical tubulovesicular compartment (TVC), but the lipid droplets (*) were devoid of labelling. Bars $0.5 \mu \mathrm{m}$.

which directly interacts with the receptor. ${ }^{41}$ Conceivably, SRBI may function as an endocytic or non-endocytic receptor, depending on cell type and the ligand, but clearly more work is needed to solve this question.
In the plasma membrane of $\mathrm{CHO}$ cells, SR-BI has previously been reported to reside in caveolae and to engage in cholesterol uptake that is clathrin coated pit independent. ${ }^{36}$ Furthermore, the subsequent transport of cholesterol out of 
A

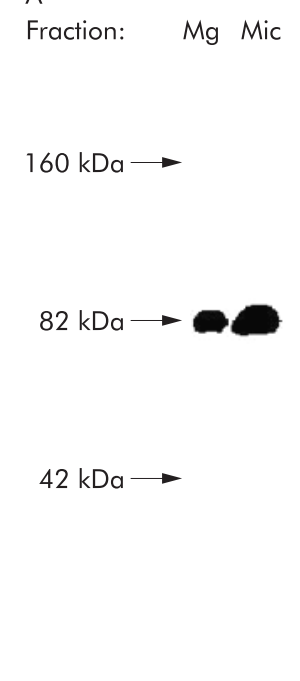

SR-BI
Mg Mic Sol

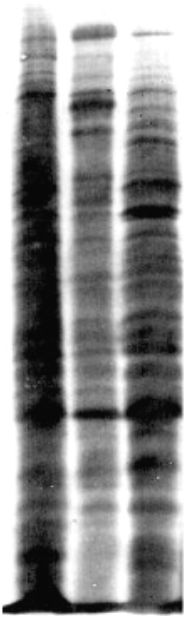

Protein
B

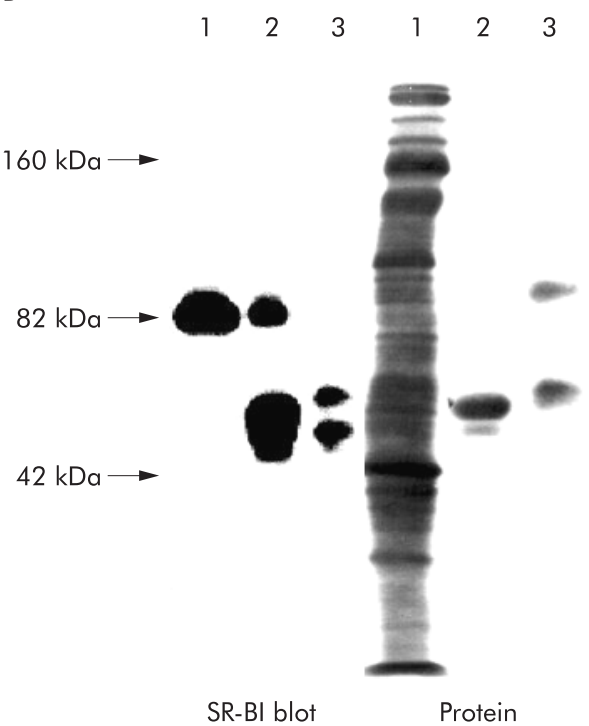

Figure 5 Subcellular localisation of scavenger receptor class B type I (SR-BI) in enterocytes. (A) Small intestinal mucosa was fractionated into $\mathrm{Mg}^{2+}$ precipitated membranes $(\mathrm{Mg})$, microvillar membranes (Mic), and soluble protein (Sol), as described in the methods section. Samples of the three subcellular fractions proportional to their relative amount in the homogenate (about 50-200 $\mu \mathrm{g}$ of protein) were subjected to sodium dodecyl sulphatepolyacrylamide gel electrophoresis. After electrophoresis and transfer onto Immobilon, the $82 \mathrm{kDa}$ band of SR-BI was visualised by immunoblotting After blotting, total protein was stained by Coomassie brilliant blue. (B) Immunopurification of SR-BI. Microvillar membranes (1) were solubilised by $1 \%$ Triton X-100 at $20^{\circ} \mathrm{C}$ (both raft and non-raft membranes are solubilised at this temperature), and preincubated with protein A-Sepharose, followed either by anti-SR-BI antibodies coupled to protein A-Sepharose (2) or free protein A-Sepharose (3), as described in the methods section. Samples were analysed by immunoblotting as described above. (The broad band of $50 \mathrm{kDa}$ in lane 2 represents the SR-BI antibody. In lane 3, protein A-Sepharose bound some endogeneous immunoglobulin and secretory component.)

A

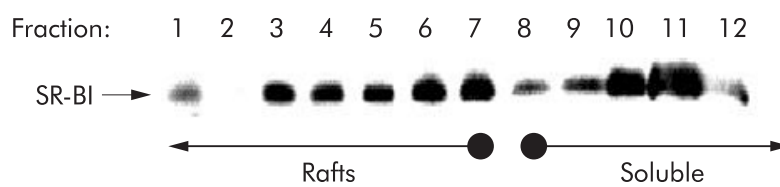

B

$\begin{array}{lllllllllllll}\text { Fraction: } & 1 & 2 & 3 & 4 & 5 & 6 & 7 & 8 & 9 & 10 & 11 & 12\end{array}$

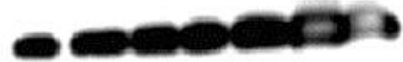

Gal-4 $\rightarrow=0$

Lactase $\longrightarrow$

Rafts

Figure 6 Lipid raft analysis of scavenger receptor class B type I (SR-BI). Sucrose gradient centrifugation of $\mathrm{Mg}^{2+}$ precipitated $(\mathrm{A})$ and microvillar (B) membranes prepared from the same mucosal homogenate. The membranes were prepared, extracted with $1 \%$ ice cold Triton X-100, and subjected to sucrose gradient centrifugation, as described in the methods section. Samples of the gradient fractions were analysed by sodium dodecyl sulphate-polyacrylamide gel

electrophoresis and immunoblotting for the distribution of SR-BI and, in the case of the microvillar gradient, for the lipid raft marker galectin-4 (Gal-4) and the "non-raft" marker lactase-phlorizin hydrolase. Gradient fractions containing lipid raft membranes and soluble ("non-raft") proteins are indicated by arrows.

caveolae into the cell reportedly occurs in a complex with caveolin and annexin A2. ${ }^{10}$ However appealing to us, such a mechanism of internalisation is unlikely to play a major role

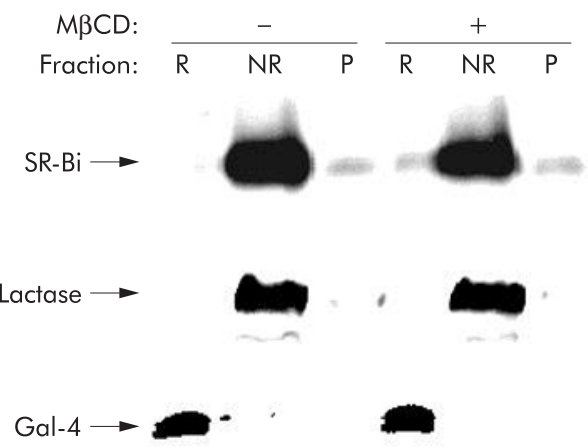

Figure 7 "Non-raft" localisation of microvillar scavenger receptor class B type I (SR-BI) is not cholesterol dependent. Microvillar membranes were resuspended in $25 \mathrm{mM}$ HEPES-HCl, $150 \mathrm{mM} \mathrm{NaCl}, \mathrm{pH} 7.1$, in the presence or absence of $2 \%$ methyl- $\beta$-cyclodextrin $(M B C D)$ for 30 minutes at room temperature before cooling on ice and solubilisation by $1 \%$ Triton X-100 for 10 minutes. After centrifugation at $20000 \mathrm{~g}$

30 minutes, the supernatant (NR, the "non-raft" fraction) was collected, and the pellet was resuspended in the above buffer and solubilised by $1 \%$ Triton X-100 for 10 minutes at $37^{\circ} \mathrm{C}$. After centrifugation as above, the resulting supernatant $(R$, the lipid raft fraction) and pellet $(P$, insoluble cytoskeletal proteins) were collected. NR, $R$, and $P$ samples were subjected to sodium dodecyl sulphate-polyacrylamide gel electrophoresis. After electrophoresis, SR-BI, lactase, and galectin-4 (Gal-4) were detected by immunoblotting.

for brush border SR-BI in the enterocyte for the following reasons. Morphologically, few if any caveolae are localised at the enterocyte brush border, despite the fact that noncaveolar lipid raft membrane microdomains are abundant at the apical surface of this cell type, ${ }^{22}$ including some pleiomorphic invaginations termed deep apical tubules between adjacent microvilli. ${ }^{42}$ Alternatively, a non-caveolar, yet lipid raft based mechanism of internalisation might be responsible for SR-BI internalisation from the brush border, 
but the observation that SR-BI from the microvillar fraction was not lipid raft associated argues against it. Although participation of a lipid raft based mechanism cannot be ruled out, the clathrin coated invaginations/pits harbouring the receptor during fat absorption implied this mechanism of internalisation to be active in endocytosis of SR-BI from the brush border. We may speculate that partial lipid raft localisation of SR-BI in $\mathrm{Mg}^{2+}$ precipitated membranes suggests that the receptor, after endocytic trafficking, encounters a lipid raft compartment and becomes part of this microdomain environment. A likely place where this might occur could well be the subapical tubulovesicular compartment which plays a central role as a sorting centre in apical membrane trafficking ${ }^{43}$ and which has previously been shown to contain IgA positive lipid raft microdomains in the enterocyte. $^{32}$ The subsequent transport to cytosolic lipid droplets, the likely final destination of SR-BI following endocytosis, may well be mediated by a lipid raft based mechanism. Alternatively, the SR-BI may be recycled from the tubulovesicular compartment to the brush border by such a lipid raft dependent mechanism.

To date, physiological ligands other than cholesterol remain to be identified for enterocyte SR-BI. Hypothetically, any lipophilic dietary/biliary constituent could be a relevant ligand in the subsequent trafficking to cytoplasmic lipid droplets. Triacylglycerol synthesis occurs in the membranes of the endoplasmic reticulum ${ }^{44}{ }^{45}$ and nascent SR-BI positive droplets could be seen at this subcellular site 15-30 minutes after the beginning of fat absorption. As chylomicrons, characterised by labelling with apolipoprotein A-1 antibody, were largely free of SR-BI, the receptor remains within the cell during subsequent export of lipoproteins, as also indicated by SR-BI labelling of small remnant lipid droplets seen in the fasting state. Lipid droplets are dynamic cytoplasmic organelles, ${ }^{44}$ and in other cell types specific targeting of proteins to these structures has been described. Thus in adipocytes, perilipins and adipose differentiation related protein localise to lipid droplets, ${ }^{46}$ and in HepG2 cells, caveolin-2, but not caveolin-1, is confined to lipid droplets. ${ }^{47}$ Whether SR-BI exerts a specific function in enterocyte lipid droplets is an interesting question that must await further work. Although its role as a physiological HDL receptor is well established, it has also been recognised that this multiligand scavenger receptor may function in other as yet undefined activities. For example, it has been speculated that multiligand lipoprotein receptors such as SR-BI evolved as pattern recognition receptors for host defence purposes prior to more specialised receptors, such as the LDL receptor. ${ }^{15}$ It is also possible that SR-BI, by way of interaction via its cytosolic PDZ domain, is in functional proximity with other brush border proteins, be it enzymes or receptors. Hopefully, the enigmas still relating to SR-BI functioning in the intestine will be solved by future work.

\section{ACKNOWLEDGEMENTS}

This work was supported by grants from the Danish Medical Research Council and the Novo-Nordic Foundation, and was part of the Danish Biotechnology programme.

\section{Authors' affiliations}

G H Hansen, L-L Niels-Christiansen, L Immerdal, E M Danielsen, Department of Medical Biochemistry and Genetics, Panum Institute, University of Copenhagen, Blegdamsvej 3, DK-2200 Copenhagen N, Denmark

\section{REFERENCES}

1 Krieger M. Charting the fate of the "good cholesterol": identification and characterization of the high-density lipoprotein receptor SR-BI. Annu Rev Biochem 1999:68:523-58.
2 Krieger M, Stern DM. Series introduction: multiligand receptors and human disease. J Clin Invest 2001;108:645-7.

3 Silver DL, Tall AR. The cellular biology of scavenger receptor class B type I. Curr Opin Lipidol 2001;12:497-504.

4 Acton SL, Scherer PE, Lodish HF, et al. Expression cloning of SR-BI, a CD36 related class B scavenger receptor. J Biol Chem 1994;269:21003-9.

5 Trigatti BL, Rigotti A, Braun A. Cellular and physiological roles of SR-BI, a lipoprotein receptor which mediates selective lipid uptake. Biochim Biophys Acta 2000; 1529:276-86.

6 Trigatti B, Rigotti A, Krieger M. The role of the high-density lipoprotein receptor SR-BI in cholesterol metabolism. Curr Opin Lipidol 2000;1 1:123-31.

7 Rodrigueza WV, Thuahnai ST, Temel RE, et al. Mechanism of scavenger receptor class B type I-mediated selective uptake of cholesteryl esters from high density lipoprotein to adrenal cells. J Biol Chem 1999;274:20344-50.

8 Babitt J, Trigatti B, Rigotti A, et al. A high density lipoprotein receptor that mediates selective lipid uptake, is $\mathrm{N}$-glycosylated and fatty acylated and colocalizes with plasma membrane caveolae. J Biol Chem 1997:272:13242-9.

9 Graf GA, Matveev SV, Smart EJ. Class B scavenger receptors, caveolae and cholesterol homeostasis. Trends Cardiovasc Med 1999;9:221-5.

10 Uittenbogaard A, Everson WV, Matveev SV, et al. Cholesteryl ester is transported from caveolae to internal membranes as part of a caveolinannexin II lipid-protein complex. J Biol Chem 2002;277:4925-31.

11 Silver DL, Wang N, Xiao X, et al. High density lipoprotein (HDL) particle uptake mediated by scavenger receptor class B type 1 results in selective sorting of $\mathrm{HDL}$ cholesterol from protein and polarized cholesterol secretion. $J$ Biol Chem 2001;276:25287-93.

12 Hauser H, Dyer JH, Nandy A, et al. Identification of a receptor mediating absorption of dietary cholesterol in the intestine. Biochemistry 1998:37:17843-50.

13 Lobo MV, Huerta L, Ruiz-Velasco N, et al. Localization of the lipid receptors CD36 and CLA-1/SR-BI in the human gastrointestinal tract: towards the identification of receptors mediating the intestinal absorption of dietary lipids. J Histochem Cytochem 2001;49:1253-60.

14 Cai SF, Kirby RJ, Howles PN, et al. Differentiation-dependent expression and localization of the class B type I scavenger receptor in intestine. J Lipid Res 2001;42:902-9.

15 Krieger $M$. Scavenger receptor class B type I is a multiligand HDL receptor that influences diverse physiologic systems. J Clin Invest 2001;108:793-7.

16 Schulthess G, Compassi S, Werder M, et al. Intestinal sterol absorption mediated by scavenger receptors is competitively inhibited by amphipathic peptides and proteins. Biochemistry 2000:39:12623-31.

17 Werder $\mathrm{M}$, Han $\mathrm{CH}$, Wehrli $\mathrm{E}$, et al. Role of scavenger receptors SR-BI and CD36 in selective sterol uptake in the small intestine. Biochemistry 2001;40:11643-50.

18 Danielsen EM, Hansen GH, Poulsen MD. Apical secretion of apolipoproteins from enterocytes. J Cell Biol 1993:120:1347-56.

19 Danielsen EM, Sjostrom H, Noren O, et al. Biosynthesis of intestinal microvillar proteins. Characterization of intestinal explants in organ culture and evidence for the existence of pro-forms of the microvillar enzymes. Biochem J 1982;202:647-54.

20 Hansen GH, Wetterberg LL, Sjostrom $\mathrm{H}$, et al. Immunogold labelling is a quantitative method as demonstrated by studies on aminopeptidase $\mathrm{N}$ in microvillar membrane vesicles. Histochem J 1992;24:132-6.

21 Danielsen EM, Hansen GH, Niels-Christiansen LL. Localization and biosynthesis of aminopeptidase $\mathrm{N}$ in pig fetal small intestine. Gastroenterology 1995; 109:1039-50.

22 Hansen GH, Niels-Christiansen LL, Thorsen E, et al. Cholesterol depletion of enterocytes. Effect on the Golgi complex and apical membrane trafficking. J Biol Chem 2000;275:5136-42.

23 Booth AG, Kenny AJ. A rapid method for the preparation of microvilli from rabbit kidney. Biochem J 1974;142:575-81.

24 Danielsen EM. Biosynthesis of intestinal microvillar proteins. Pulse-chase labelling studies on aminopeptidase $\mathrm{N}$ and sucrase-isomaltase. Biochem J 1982;204:639-45.

25 Brown DA, Rose JK. Sorting of GPI-anchored proteins to glycolipid-enriched membrane subdomains during transport to the apical cell surface. Cell 1992;68:533-44.

26 Danielsen EM. Involvement of detergent-insoluble complexes in the intracellular transport of intestinal brush border enzymes. Biochemistry 1995; 34:1596-605.

27 Laemmli UK. Cleavage of structural proteins during the assembly of the head of bacteriophage T4. Nature 1970;227:680-5.

28 van lizendoorn SC, Maier O, Van Der Wouden JM, et al. The subapical compartment and its role in intracellular trafficking and cell polarity. J Cell Physiol 2000;184:151-60.

29 Strauss EW. Morphological aspects of triglyceride absorption. In: Code CF, ed. Handbook of physiology 6. Washington, DC: American Physiological Society, 1968:1377-406.

30 Sabesin SM, Frase S. Electron microscopic studies of the assembly, intracellular transport, and secretion of chylomicrons by rat intestine. J Lipid Res 1977; 18:496-511

31 Magun AM, Mish B, Glickman RM. Intracellular apoA-I and apoB distribution in rat intestine is altered by lipid feeding. J Lipid Res 1988;29:1107-16.

32 Hansen GH, Niels-Christiansen LL, Immerdal L, et al. Transcytosis of immunoglobulin $A$ in the mouse enterocyte occurs through glycolipid raft- and rab17-containing compartments. Gastroenterology 1999;1 16:610-22.

33 Hansen GH, Immerdal L, Thorsen E, et al. Lipid rafts exist as stable cholesterolindependent microdomains in the brush border membrane of enterocytes. J Biol Chem 2001;276:32338-44. 
34 Braccia A, Villani M, Immerdal L, et al. Microvillar membrane microdomains exist at physiological temperature: Galectin-4's role as lipid raft stabilizer revealed by "superrafts". J Biol Chem 2003;278:15679-84.

35 Anderson RG. The caveolae membrane system. Annu Rev Biochem 1998:67:199-25.

36 Graf GA, Connell PM, van der Westhuyzen DR, et al. The class B, type I scavenger receptor promotes the selective uptake of high density lipoprotein cholesterol ethers into caveolae. J Biol Chem 1999;274:12043-8.

37 Thurnhofer $\mathbf{H}$, Schnabel J, Betz $M$, et al. Cholesterol-transfer protein located in the intestinal brush-border membrane. Partial purification and characterization. Biochim Biophys Acta 1991;1064:275-86.

38 Thurnhofer H, Hauser $\mathrm{H}$. Uptake of cholesterol by small intestinal brush border membrane is protein-mediated. Biochemistry 1990;29:2142-8.

39 Detmers PA, Patel S, Hernandez M, et al. A target for cholesterol absorption inhibitors in the enterocyte brush border membrane. Biochim Biophys Acta 2000;1486:243-52.

40 Mardones P, Quinones V, Amigo L, et al. Hepatic cholesterol and bile acid metabolism and intestinal cholesterol absorption in scavenger receptor class $B$ type I-deficient mice. J Lipid Res $2001 ; 42: 170-80$.
41 Ikemoto $M$, Arai $H$, Feng D, et al. Identification of a PDZ-domain-containing protein that interacts with the scavenger receptor class B type I. Proc Natl Acad Sci U S A 2000;97:6538-43.

42 Hansen GH, Pedersen J, Niels-Christiansen LL, et al. Deep apical tubules: Dynamic lipid raft microdomains in the brush border region of enterocytes. Biochem J 2003;373:125-32.

43 van lizendoorn SC, Hoekstra D. The subapical compartment: a novel sorting centre? Trends Cell Biol 1999;9:144-9.

44 Murphy DJ, Vance J. Mechanisms of lipid-body formation. Trends Biochem Sci 1999:24:109-15.

45 Hussain MM. A proposed model for the assembly of chylomicrons. Atherosclerosis 2000;148:1-15.

46 Londos C, Brasaemle DL, Schultz CJ, et al. Perilipins, ADRP, and other proteins that associate with intracellular neutral lipid droplets in animal cells. Semin Cell Dev Biol 1999;10:51-8

47 Fujimoto T, Kogo H, Ishiguro K, et al. Caveolin-2 is targeted to lipid droplets, a new "membrane domain" in the cell. J Cell Biol 2001;152:1079-85.

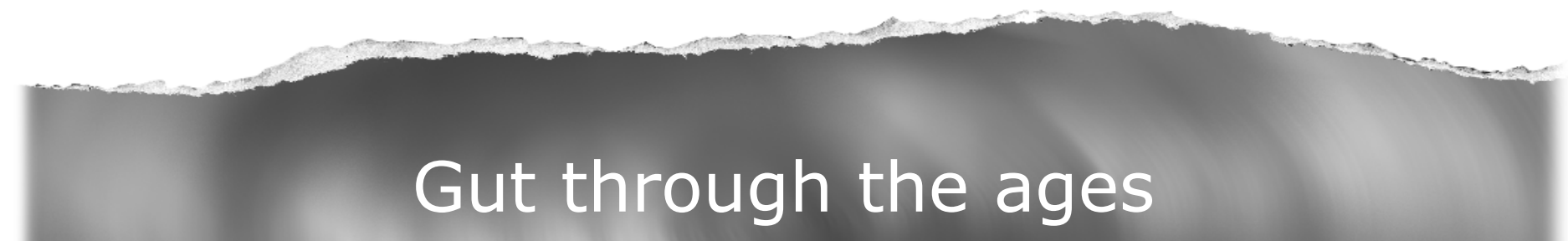

Browse the Archive

Gut online has an archive of content dating back to 1966 .

Full text from 1997; abstracts from 1975; table of contents from 1966

www.gutjnl.com 